\title{
Non-Boussinesq Integral Model for Horizontal Turbulent Buoyant Round Jets
}

\author{
J. Xiao, ${ }^{1}$ J.R. Travis, ${ }^{2}$ and W. Breitung ${ }^{1}$ \\ ${ }^{1}$ Institute for Nuclear and Energy Technologies, Forschungszentrum Karlsruhe, P.O. Box 3640, 76021 Karlsruhe, Germany \\ ${ }^{2}$ Dubois, Pitzer, Travis GmbH, Offenbach, Germany \\ Correspondence should be addressed to J. Xiao, jianjun.xiao@iket.fzk.de
}

Received 19 November 2008; Accepted 25 May 2009

Recommended by Xu Cheng

Horizontal buoyant jet is a fundamental flow regime for hydrogen safety analysis in power industry. The purpose of this study is to develop a fast non-Boussinesq engineering model the horizontal buoyant round jets. Verification of this integral model is established with available experimental data and comparisons over a large range of density variations with the CFD codes GASFLOW. The model has proved to be an efficient engineering tool for predicting horizontal strongly buoyant round jets.

Copyright () 2009 J. Xiao et al. This is an open access article distributed under the Creative Commons Attribution License, which permits unrestricted use, distribution, and reproduction in any medium, provided the original work is properly cited.

\section{Introduction}

Turbulent buoyant jet is a fundamental flow regime in hydrogen safety analysis since it affects hydrogen distribution and mitigation measures when accidents occur [1]. Recent works on the numerical simulation of helium and steam jet release experiments in the German THAI facility have shown that the Helium distribution (simulating hydrogen) may depend sensitively on the jet modeling [2]. A systematic investigation was therefore started on numerical modeling of horizontal turbulent buoyant jets [3].

Few experimental data and calculations on horizontal turbulent buoyant jet with large density variation can be found in the open literatures. Most of the experiments were carried out for the small density variation when the Boussinesq approximation is valid. Pantokratoras [4] modified the integral Fan-Brooks model [5] to calculate the horizontal penetration of inclined thermal buoyant water jets, and the modified model predictions are in a good agreement with the trajectory measurements. Jirka $[6,7]$ formulated integral model, namely, CorJet for turbulent buoyant jets (round and planar) in unbounded stratified flows, that is, the pure jet, the pure plume, the pure wake. Guerra [8] simulated horizontal buoyant jets $(64 \%$ helium into air with various inlet velocity) using CFD code FLOVENT, but unfortunately his work is only from calculation results and he did not provide any experiment data for this case.
Swain $[9,10]$ studied horizontal round hydrogen jets and determined the maximum horizontal distance at which a horizontal hydrogen leak can be ignited. Houf and Schefer [11] developed a model to study the small leaks of hydrogen in the Froude number range where both buoyant and inertial forces are important.

In this study non-Boussinesq integral model for horizontal buoyant round jet was derived with the modified entrainment hypothesis. The system of conservation equations of the integral model was solved by a forth order RungeKutta method to obtain numerical solutions in the transition region from jet-like to plume-like.

The problem description and modeling efforts are presented in Sections 2 and 3. Sections 4 and 5 documents the validation and modeling uncertainties of the integral model. The concluding remarks are given in Section 6.

\section{Problem Description}

The horizontal buoyant jet formed from a round orifice is discharged into the unbounded stagnant uniform ambient, as shown in Figure 1. The density of the ambient is $\rho_{a}$. The axis of the jet is taken as a parametrical coordinate $s$, and the coordinate $n$ is taken to be normal to the axis s. $\theta$ is the angle of $s$-axis with the horizontal direction. The initial density, velocity, and diameter in the orifice are $\rho_{0}, U_{0}, r_{0}$. The density and velocity along the $s$-axis are $\rho_{s}, u_{s}$. 


\section{Formulation of Horizontal Buoyant Jets}

3.1. Basic Governing Equations. In this study, the pressure across the flow is assumed to be uniform and equal to the ambient pressure outside of the boundary. The basic governing equations (neglecting the dissipation and turbulent transport in comparison with the mean flow) consist of mass, momentum, energy, and concentration conservation equations,

$$
\begin{gathered}
\nabla \cdot(\rho \vec{u})=0, \\
\frac{\partial\left(\rho u u_{i}\right)}{\partial x}=0, \\
\frac{\partial\left(\rho u u_{j}\right)}{\partial y}=\Delta \rho g, \\
\nabla \cdot(\rho \vec{u} h)=0, \\
\nabla \cdot\left(\rho \vec{u} \phi_{m}\right)=0,
\end{gathered}
$$

where $h$ is the specific enthalpy and $\Phi_{m}$ is the mass concentration.

The divergence theorem is applied, and the basic governing equations become

$$
\begin{gathered}
\frac{d}{d s} \int_{0}^{2 \pi} \int_{0}^{\infty}(\rho u) r d r d \varphi=2 \pi \alpha_{j-p} b \rho_{a} u_{s}=E_{m} \\
\frac{d}{d s} \int_{0}^{2 \pi} \int_{0}^{\infty}(\rho u u \cos \theta) r d r d \varphi=0 \\
\frac{d}{d s} \int_{0}^{2 \pi} \int_{0}^{\infty}(\rho u u \sin \theta) r d r d \varphi=\int_{0}^{2 \pi} \int_{0}^{\infty}\left(\rho_{a}-\rho\right) g r d r d \varphi \\
\frac{d}{d s} \int_{0}^{2 \pi} \int_{0}^{\infty}\left[\rho u\left(C_{P} T-C_{P a} T_{a}\right)\right] r d r d \varphi=0 \\
\frac{d}{d s} \int_{0}^{2 \pi} \int_{0}^{\infty}\left(\rho u \phi_{m}\right) r d r d \varphi=0 \\
\frac{d x}{d s}=\cos \theta \\
\frac{d y}{d s}=\sin \theta
\end{gathered}
$$

where $E_{m}$ is the local mass entrainment rate and $\alpha_{j-p}$ is the local mass entrainment coefficient.

A system of first-order ordinary differential equations was obtained after the integration, where the seven unknowns are the density, velocity, temperature along the trajectory, $\rho_{s}, u_{s}, T_{s}$, the characteristic jet width $b$, the local angle of the jet with respect to the horizontal axis $\theta$, and the local coordinates of the jet trajectory $x, y$. With initial conditions, the system of ordinary differential equations was solved by aforth order Runga-Kutta method to obtain the horizontal buoyant jet trajectory, the velocity, the density, the temperature and the tracer concentration.

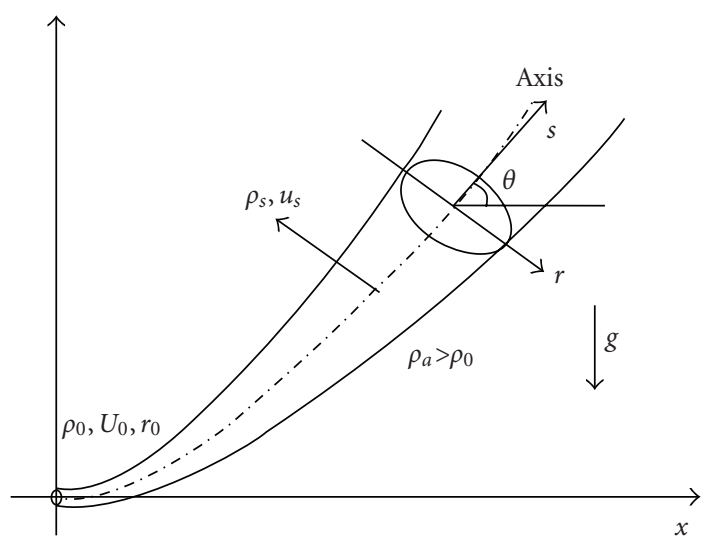

FIGURE 1: Definition diagram for horizontal buoyant jet discharges from round orifice into the unstratified ambient.

3.2. Basic Assumptions. The general assumptions made in this investigation are as follows.

(1) The flow is fully turbulent which means there is no Reynold number dependence.

(2) The profiles of velocity, density, and temperature are similar at all cross-sections normal to the jet trajectory.

(3) Longitudinal turbulent transport is small compared with latitudinal convective transport.

3.3. Profiles of Velocity, Density, and Temperature. Velocity profile is assumed to be Gaussian distribution:

$$
u=u_{s} e^{-r^{2} / b^{2}}
$$

where $b$ is a characteristic jet width.

Density deficiency profile with respect to the ambient density in a uniform ambient is assumed to be Gaussian:

$$
\frac{\rho_{a}-\rho}{\rho_{a}}=\left(\frac{\rho_{a}-\rho_{s}}{\rho_{a}}\right) e^{-r^{2} /(\lambda b)^{2}},
$$

where $\lambda b$ is the characteristic length of the profiles; $\lambda^{2}$ is the turbulent Schmidt number, which is assumed to be constant and is usually found to be somewhat larger than 1 for small density ratio cases. In this study $\lambda$ is 1.2 . It should be noted that $\lambda^{2}$ is not necessarily constant as the buoyant jet evolves from jet-like to plume-like.

Due to the large density or temperature variation between the jet and the ambient considered in the nonBoussinesq model, the density in the trajectory $\rho_{s}$ is the function of temperature $T_{s}$ and mass faction $\Phi_{m s}$. Therefore, the energy equation should be solved. It is well known that the cross-sectional profiles of normalized mean axial velocity and concentration are Gaussian-like in the zone of established flow (ZEF). In this study, the profile of temperature is also assumed to be Gaussian distribution:

$$
\frac{T_{a}-T}{T_{a}}=\left(\frac{T_{a}-T_{s}}{T_{a}}\right) e^{-r^{2} /(\lambda b)^{2}},
$$


3.4. Entrainment Hypothesis. To close the equations system, the mass entrainment rate should be specified. The entrainment relation for the horizontal round jet is given by:

$$
E_{m}=2 \pi b \rho_{a} u_{e}=2 \pi \alpha_{j-p} b \rho_{a} u_{s}
$$

where $E_{m}$ is the local mass entrainment rate, $u_{e}$ is the local entrainment velocity, $u_{s}$ is the characteristic velocity along $s$ axis, $\rho_{s}$ is the local density along s-axis, $\rho_{a}$ is the density of ambient, and $\alpha_{j-p}$ is the local mass entrainment coefficient .

For low-momentum buoyant jets, experimental data indicates that the local rate of entrainment increases as the jets leaves the momentum-dominated region and enters a region where the effects of buoyancy become more pronounced. In Jirka's paper [6], the local rate of entrainment is specified as

$$
E_{m}=2 \pi b U_{s}\left(\alpha_{j}+\alpha_{p} \frac{F r_{p}^{2}}{F r_{s}^{2}} \sin \theta\right)
$$

List summarized much of the work on the entrainment hypothesis and proposed values of $\alpha_{j}=0.052 \pm 0.003$ for the pure jets and $\alpha_{p}=0.0833 \pm 0.0042$ for the pure plumes [12]. To model the entrainment in the region of transition from jet-like to plume-like, an empirical function for the vertical plane jet proposed,

$$
\alpha_{j-p}=\alpha_{j}+\left(\alpha_{p}-\alpha_{j}\right)\left(\frac{R i_{j-p}}{R i_{p}}\right)^{2},
$$

$R i_{j-p}$ is the local Richardson number in the trajectory which is defined as

$$
R i_{j-p}=\frac{m \beta^{1 / 2}}{m o^{5 / 4}}
$$

where $m$ is the mass flux, mo is the momentum flux, $\beta$ is the local buoyancy flux. The Richardson number in the pure plume region $R i_{p}$ is a constant.

In the non-Boussinesq model, the effect of large density variation should be considered in the entrainment coefficient. In this study the local entrainment coefficient for the horizontal buoyant jet is assumed as:

$$
\alpha_{j-p}=\left[\alpha_{j}+\left(\alpha_{p}-\alpha_{j}\right)\left(\frac{R i_{j-p}}{R i_{p}}\right)^{2} \sin \theta\right] *\left(\frac{\rho_{s}}{\rho_{a}}\right)^{1 / 2}
$$

for the round jet, the exponent is $1 / 2, \alpha_{j}=0.055$ for the pure jets and $\alpha_{p}=0.085$ for the pure plume. We should note that the value of $\alpha_{j}$ here is obtained from the flow with low velocity. For the flow with high velocity, $\alpha_{j}$ may be varied. It will be discussed in the section of modeling uncertainties.

\section{Validations}

4.1. Pure Jet. For the pure jet $\left(\theta=0^{\circ}, \rho_{a}=\rho_{0}\right)$, there is no buoyancy acting on the flow and the jet is only dominated by

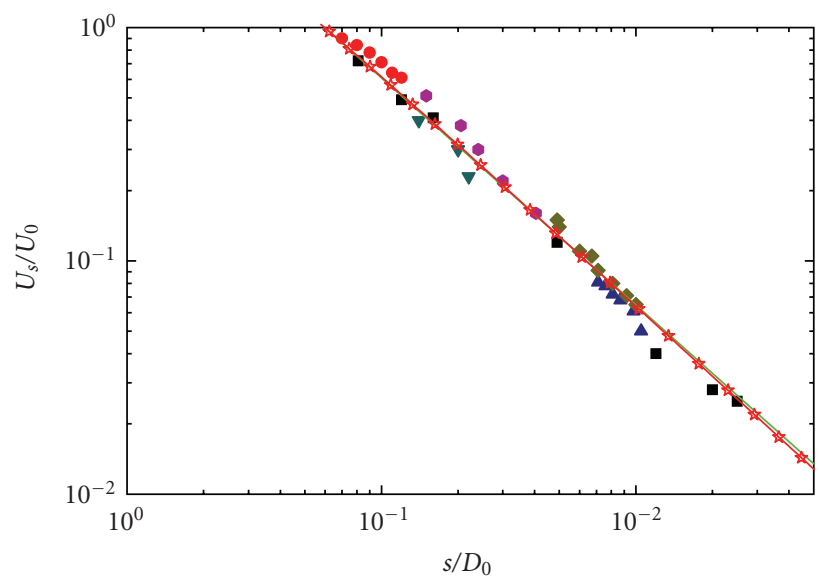

- Albertson (1950)

- Crow and Charnpagne (1971)

- Capp (1983)

$\checkmark \quad$ Labus and Symons (1971)

- Hussein (1994)

- Papanicolaou and List (1988)

- Analytical solution

↔ NB integral model

FIgURE 2: Centerline velocity decay for pure jets.

the momentum. The conservation equations of the pure jet can be expressed as:

$$
\begin{gathered}
\frac{d}{d s}\left(\pi \rho_{a} u_{s} b_{s}^{2}\right)=2 \pi \alpha_{\mathrm{jet}} \rho_{a} u_{s} b_{s}, \\
\frac{d}{d s}\left(\frac{\pi}{2} \rho_{a} u_{s}^{2} b_{s}^{2}\right)=0 \\
\frac{d}{d s}\left(\frac{\pi \lambda^{2}}{1+\lambda^{2}} c_{s} u_{s} b_{s}^{2}\right)=0 .
\end{gathered}
$$

The ratio of $u_{s}$ and the initial velocity $U_{0}$ is,

$$
\frac{u_{s}}{U_{0}}=\frac{\sqrt{2}}{4 \alpha_{j}}\left(\frac{s}{D_{0}}\right)^{-1} \text {. }
$$

The centerline dilution is,

$$
\frac{1+\lambda^{2}}{\lambda^{2}} S_{c}=\left(\sqrt{8 \pi} \alpha_{j}\right) \frac{s}{D_{0}} .
$$

The centerline velocity decay and concentration dilution are shown in Figures 2 and 3. When $\alpha_{\mathrm{jet}}$ and $\lambda$ are 0.055 and 1.2, the results of the non-Boussiensq integral model developed in this study have good agreement with the experimental data and the analytical solutions in (12) and (13).

4.2. Bouyant Jet with Small Density Variation. The horizontal buoyant jets with small density variations $(<5-10 \%)$ are predicted with the non-Boussinesq integral model. The nitrogen $(300 \mathrm{~K}, 1 \mathrm{bar})$ is injected horizontally into the ambient ( $300 \mathrm{~K}, 1$ bar) with various initial Froude numbers. Figure 4 shows the normalized vertical trajectory $Y / L_{M}$ as a function of $X / L_{M}$ for a variety of experimental conditions 


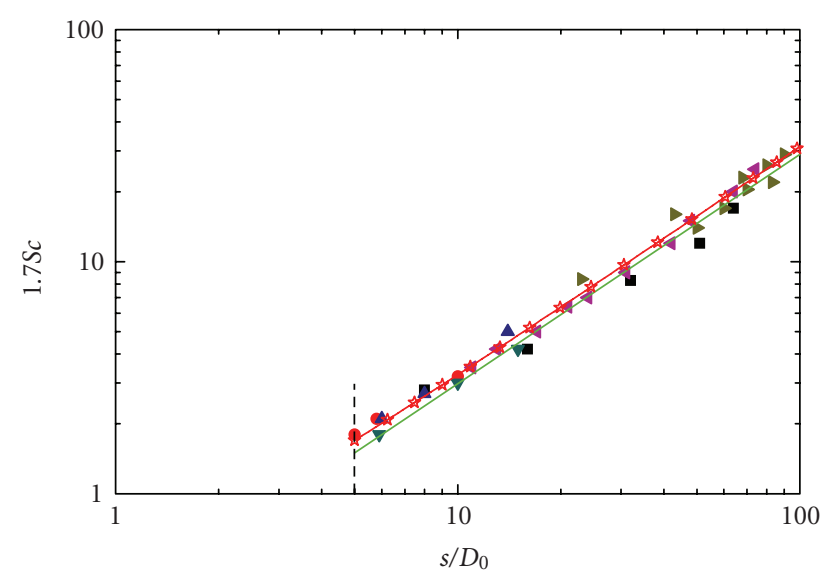

- Albertson (1950)

- Crow and Charnpagne (1971)

- Hill (1972)

- Labus and Symons (1971)

4 Ricou and Spalding (1961)

- Papanicolaou and List (1988)

— Analytical solution \& CorJet model, Jirka (2004)

\# NB integral model

Figure 3: Concentration decay along the centerline for pure jets.

and the comparisons of the non-Boussinesq integral model and the Boussinesq-based CorJet model.

The length scale $L_{M}$ for the transition of horizontal buoyant round jet to plume is defined as

$$
L_{M}=\frac{m o_{0}^{3 / 4}}{j_{0}^{1 / 2}}
$$

where

$$
m o_{0}=U_{0}^{2} A_{0}, j_{0}=U_{0}\left(\frac{\left(\rho_{a}-\rho_{0}\right) g}{\rho_{0}}\right) A_{0} .
$$

The normalized centerline dilutionSc/ $\mathrm{Fr}_{0}$ as a function of $Y / L_{M}$ is depicted in Figure 5. The Froude number is:

$$
F r_{0}=\frac{U_{0}}{\sqrt{\left(\left(\rho_{a}-\rho_{0}\right) g / \rho_{0}\right) R_{0}}} .
$$

The predictions of non-Boussinsq model agree well with the experimental data. When the initial Froude number $\mathrm{Fr}_{0}$ is high enough the non-Boussinesq model's results will collapse to the CorJet model. It indicates that in high Froude number flow the influence of small density variation can be neglected. However in the flows with low Froude numbers it seems that the effects of the buoyancy should be considered. The normalized trajectory will not collapse when the Froude number is small which means the Boussinesq approximation is invalid when the buoyancy effect is comparative with the momentum effect.

The non-Boussinesq integral model provides a satisfactory transition behavior for the horizontal buoyant jets with small density variations from the jet-like to plumelike region. Figures 6,7 , and 8 depict the predictions

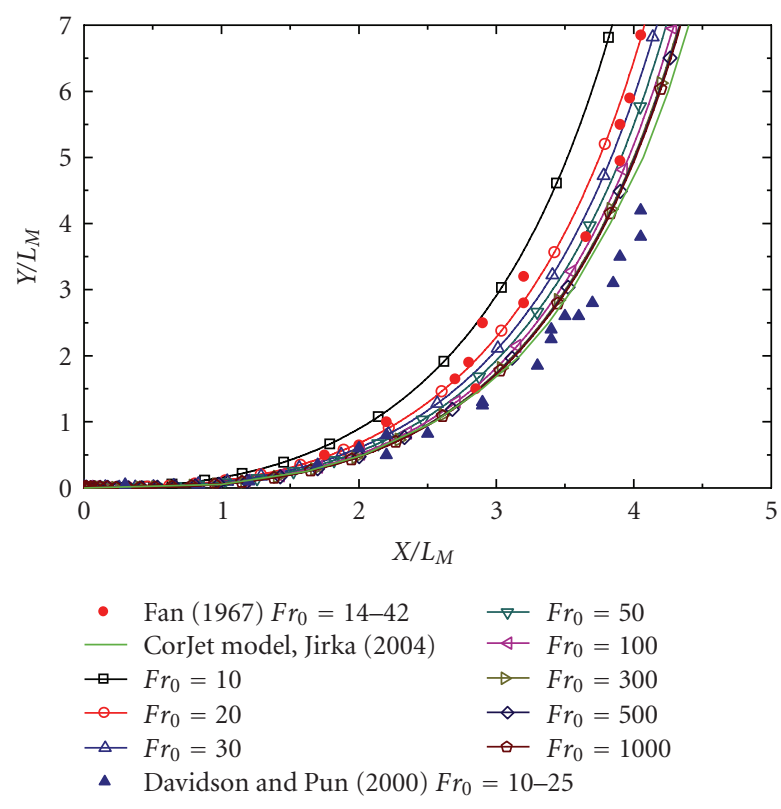

Figure 4: Normalized trajectories of horizontal buoyant jet.

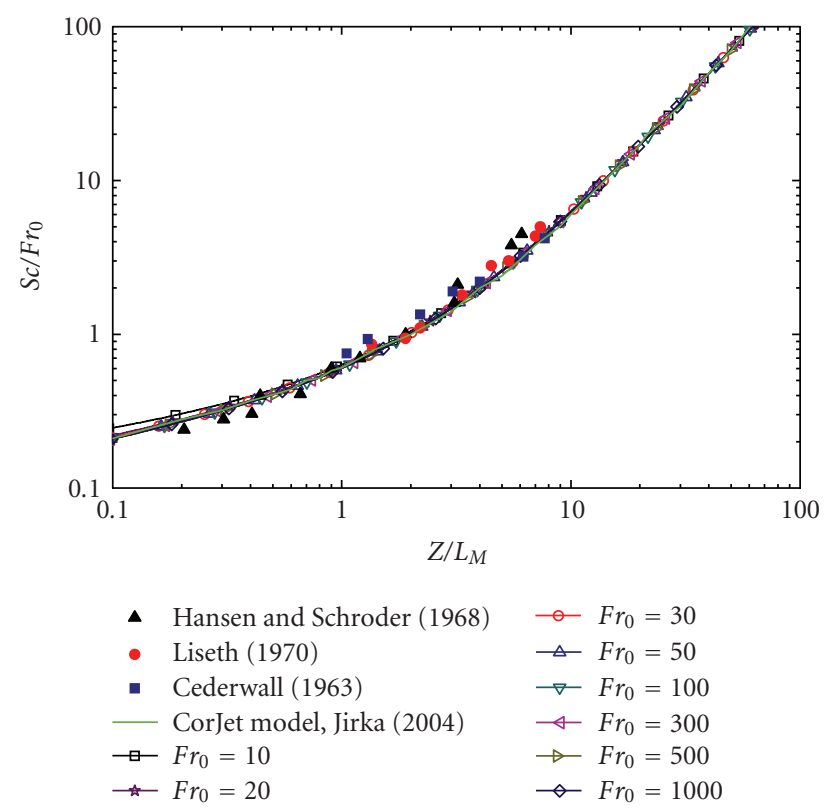

FIGURE 5: Normalized centerline dilutions of horizontal buoyant jet.

of non-Boussineq integral model: the decays of velocities, Froude numbers, and the entrainment coefficients along the centerline of the horizontal buoyant jets.

4.3. Buoyant Jet with Large Density Variation. The horizontal buoyant jets with large density variations, for instance hydrogen or helium injecting into air, have not received sufficient research before, and almost no experimental data could be found in the open literature. CFD code GASFLOW $[13,14]$ was used to validate the non-Boussinesq integral model. Helium at $300 \mathrm{~K}, 1$ bar is injected into the air $(300 \mathrm{~K}$, $1 \mathrm{bar}$ ) with various efflux velocities. The diameter of the orifice is $0.0845 \mathrm{~m}$. 


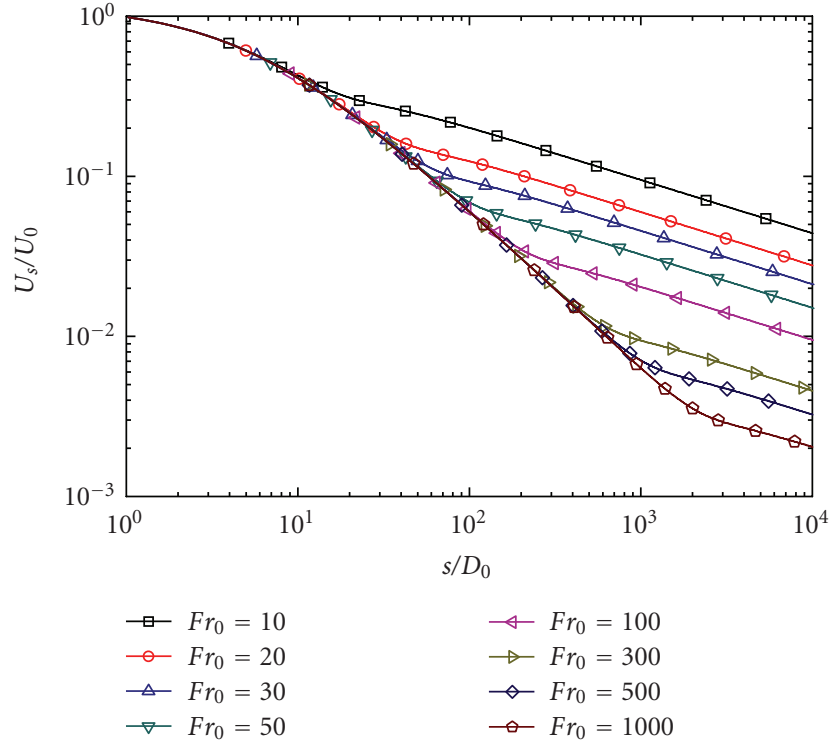

Figure 6: Velocity decay of horizontal buoyant jet.

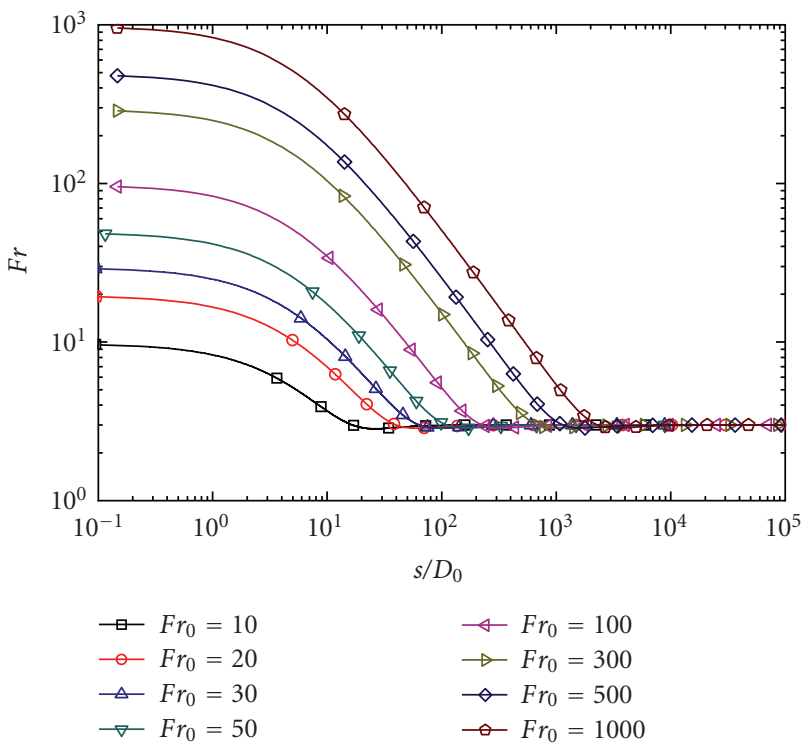

FIGURE 7: Froude number decay of horizontal buoyant jet.

Figure 9 shows the trajectories of the horizontal buoyant jets. The non-Boussinesq integral model also provides good transition behavior for the horizontal buoyant jets with large density variations. The decays of the velocity and concentration along the trajectory are shown in Figures 10 and 11. Although small discrepancies exist between the results of the integral model and CFD code GASFLOW, the nonBoussinesq integral model is capable of predicting the horizontal buoyant jets with large density variations. Experimental data are expected for the further validation of the model.

\section{Modeling Uncertainties}

Before the Gaussian profiles are reached, the initial unsheared profiles undergo changes in form of peripherally

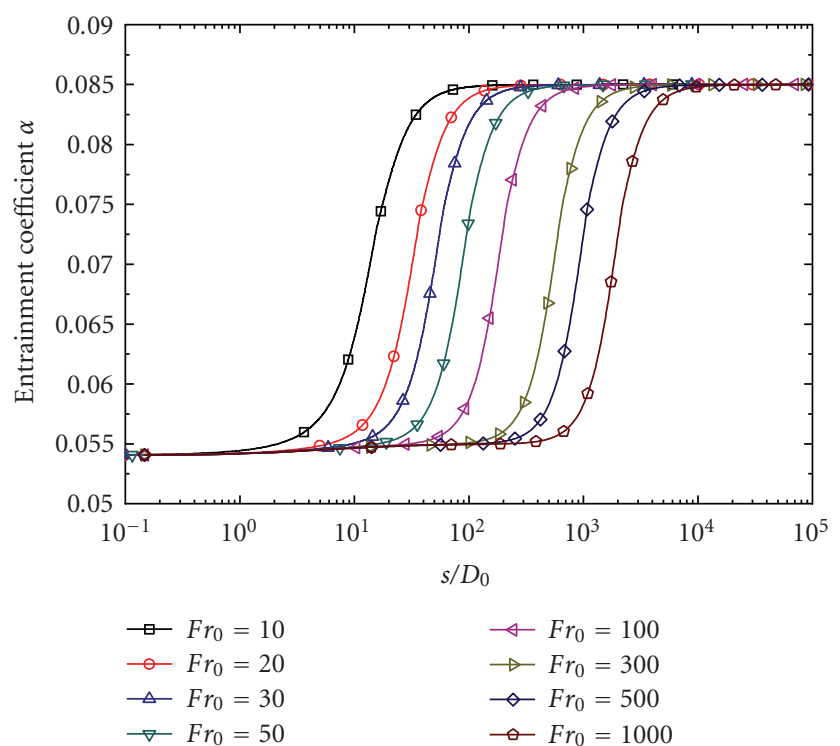

FIGURE 8: Entrainment coefficients of horizontal buoyant jet.

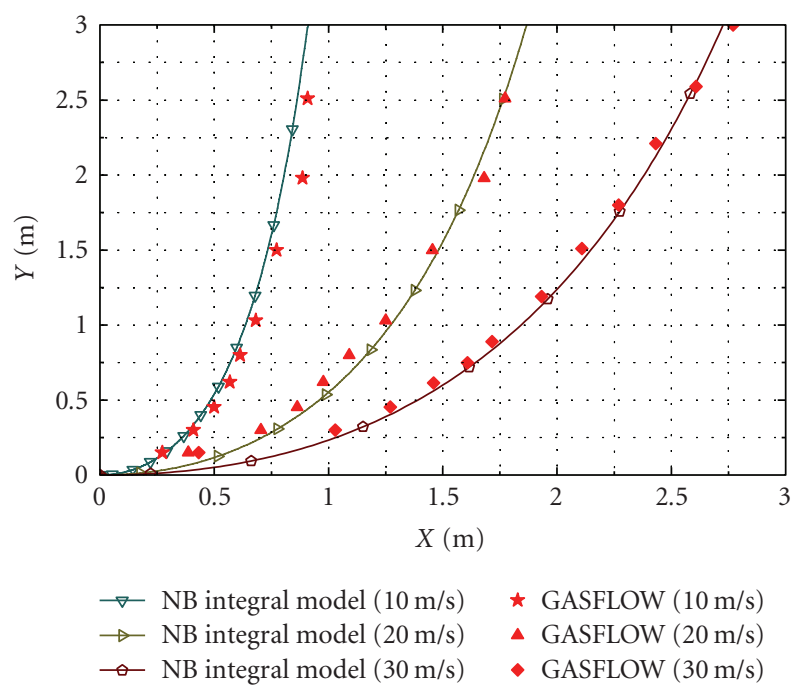

FIGURE 9: Trajectories of horizontal buoyant jet with large density variations.

growing axis symmetric mixing layers. This initial region is called the zone of flow establishment which lacks of selfsimilarity. The transition in this region is complex and rapid, and the distance is up to 5-10 diameter of the orifice. A distance of 5-10 diameters from the orifice is shifted in the study.

The mass entrainment coefficients in this study were obtained under the experimental conditions when the velocity and density variation are not so high. How the high velocity and large density variation affect the entrainment coefficient is not clear. In the recent simulation of the underexpanded hydrogen jet [15], it indicates that the entrainment coefficient for the pure jet, $\alpha_{j}$, increases from 0.055 to $0.065-0.07$ when the velocity is sonic or supersonic. 


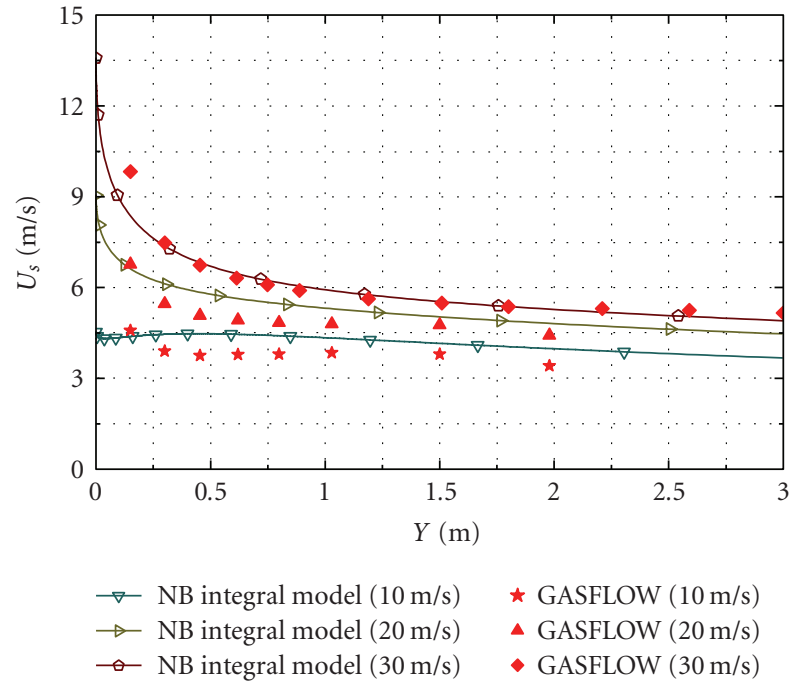

FIgURE 10: Velocity decay of horizontal buoyant jet with large density variations.

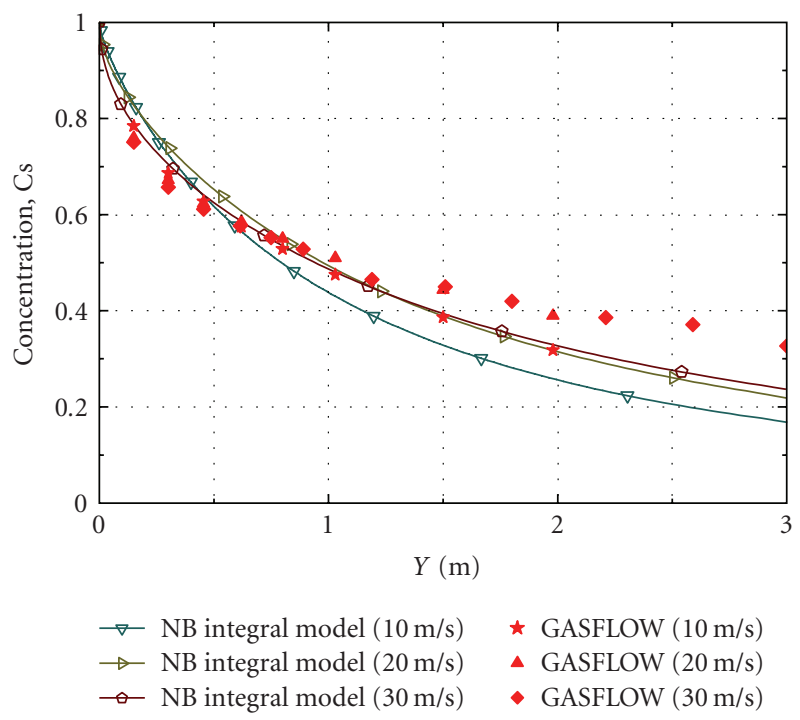

FIGURE 11: Concentration decay of horizontal buoyant jet with large density variations.

The mechanisms of these uncertainties needs further study in the future work.

\section{Concluding Remarks}

This non-Boussinesq integral model developed in the study is a fast engineering model to solve the horizontal buoyant round jets problems. The model was validated by the pure jet, horizontal buoyant jets with small/large density variations, and good agreements with the experimental data, and CFD predictions were obtained.

For strongly buoyant jet the Boussinesq approximation is violated which will over-predict the mass entrainment and under-estimate the buoyancy effect [16]. It reveals that the Boussinesq approximation is valid when the density variation is less than $10 \%$.

The entrainment assumption is a key requirement for the integral model. The entrainment assumption taking into account the Richard number and the angle $\theta$ was used in the integral model, and satisfactory predictions for the modeling of horizontal buoyant jets were provided. The effects of large density variation and high velocity on the entrainment coefficient need further study in the future.

\section{References}

[1] W. Breitung, "State of the art report on containment thermal hydraulics and hydrogen distribution," OECD/NEA, 1999.

[2] P. Royl, U. J. Lee, J. R. Travis, and W. Breitung, "Benchmark of the 3D CFD code GADFLOW II with containment thermal hydraulic tests from HDR and THAI," in CFD4NRS Conference, Munich, Germany, September 2006.

[3] J. Xiao, J. Travis, and W. Breitung, "Numerical simulation of free horizontal turbulent buoyant planar jets and plumes," in Proceedings of the Annual Meeting on Nuclear Technology, Karlsruhe, Germany, May 2007.

[4] A. Pantokratoras, "Horizontal penetration of inclined thermal buoyant water jets," International Communications in Heat and Mass Transfer, vol. 25, no. 4, pp. 561-569, 1998.

[5] L. N. Fan and N. H. Brooks, Numerical Solution of Turbulent Buoyant Jets Problems, California Institute of Technology, Pasadena, Calif, USA, 1969.

[6] G. H. Jirka, "Integral model for turbulent buoyant jets in unbounded stratified flows-part I: single round jet," Environmental Fluid Mechanics, vol. 4, no. 1, pp. 1-56, 2004.

[7] G. H. Jirka, "Integral model for turbulent buoyant jets in unbounded stratified flows-part 2: plane jet dynamics resulting from multiport diffuser jets," Environmental Fluid Mechanics, vol. 6, no. 1, pp. 43-100, 2006.

[8] D. Guerra, Modelisation du transfert d'un aerocontaminant dans un local ventile en champ proche d'une source d'emission accidentelle, Ph.D. thesis, Polytechnique of Toulouse, Toulouse, France, 2004.

[9] M. Swain, "Codes and standards analysis, 2004 annual program review meeting of the hydrogen," Fuel cell \& infrastructure program of the US DOE, 2004.

[10] M. Swain, "Hydrogen properties testing and verification," in Proceedings of the Fuel Cell Summit Meeting, Coral Gables, Fla, USA, June 2004.

[11] W. Houf and R. Schefer, "Small-scale unintended releases of hydrogen," in Proceedings of the Annual Hydrogen Conference and Hydrogen Expo, San Antonio, Tex, USA, March 2007.

[12] W. Rodi, Turbulent Buoyant Jets and Plumes, vol. 6 of HMT The Science \& Applications of Heat and Mass Transfer, Pergamon Press, Oxford, UK, 1982.

[13] J. R. Travis, J. W. Spore, P. Royl, et al., GASFLOW: A Computational Fluid Dynamics Code for Gases, Aerosols and Combustion. Volume 1: Theory and Computational Model, FzK and LANL, 2001.

[14] J. R. Travis, J. W. Spore, P. Royl, et al., GASFLOW: A Computational Fluid Dynamics Code for Gases, Aerosols and Combustion. Volume 2: User's Manual, FzK and LANL, 2001.

[15] A. Veser, G. Stern, M. Schwall, et al., "Structure and flame propagation regimes in turbulent hydrogen jets," in Proceedings of 7 th International Symposium on Hazards, Prevention, and Mitigation of Industrial Explosions, vol. 1, pp. 198-207, Saint-Petersburg, Russia, July 2008. 
[16] J. Xiao, J. Travis, and W. Breitung, "Non-boussinesq integral model for horizontal turbulent strongly buoyant plane jets," in Proceedings of the 16th International Conference on Nuclear Engineering, Orlando, Fla, USA, May 2008. 

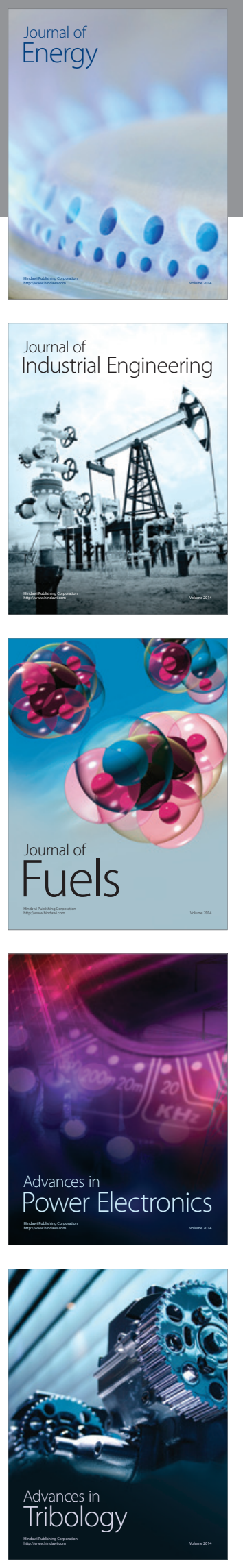
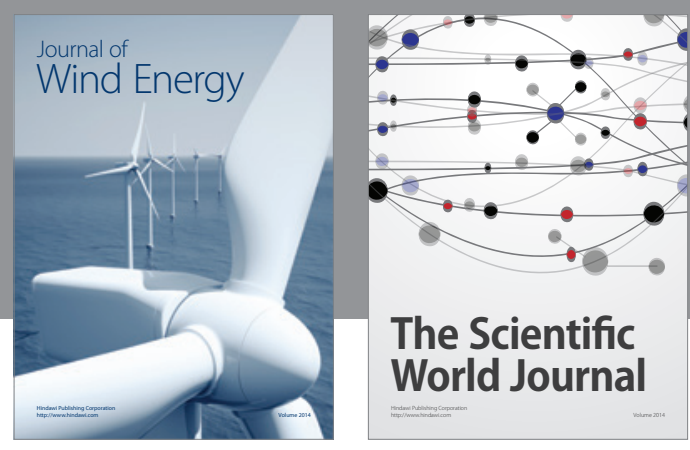

The Scientific World Journal

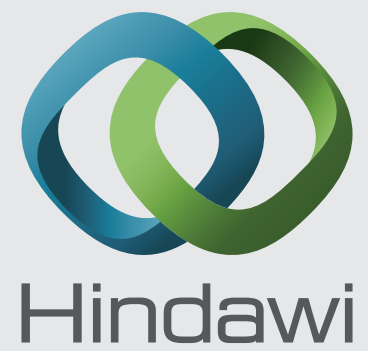

Submit your manuscripts at http://www.hindawi.com
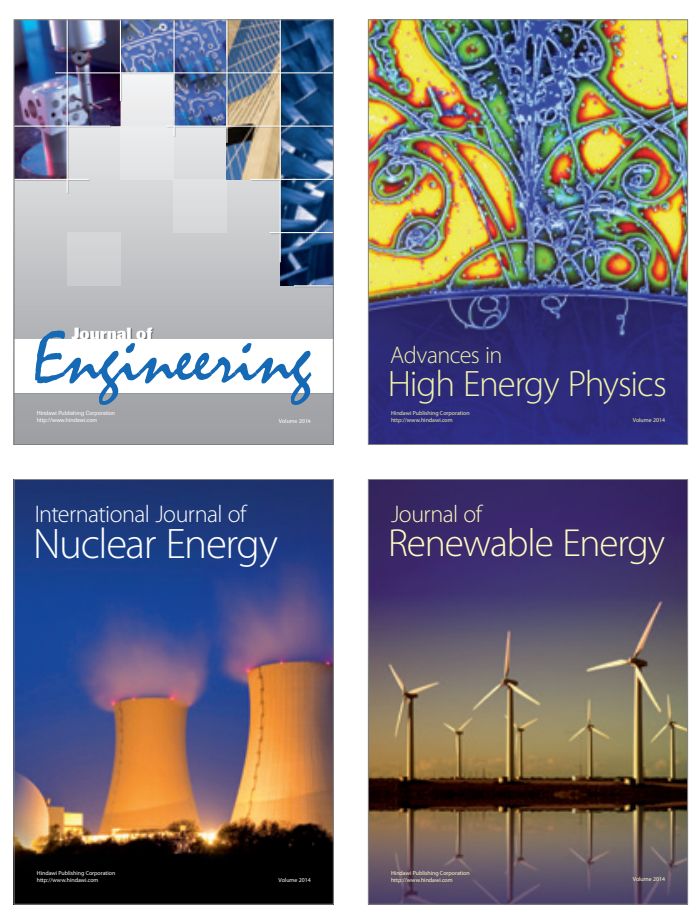

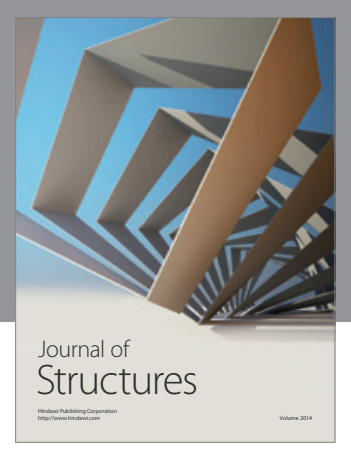

Rotating
Mechinery
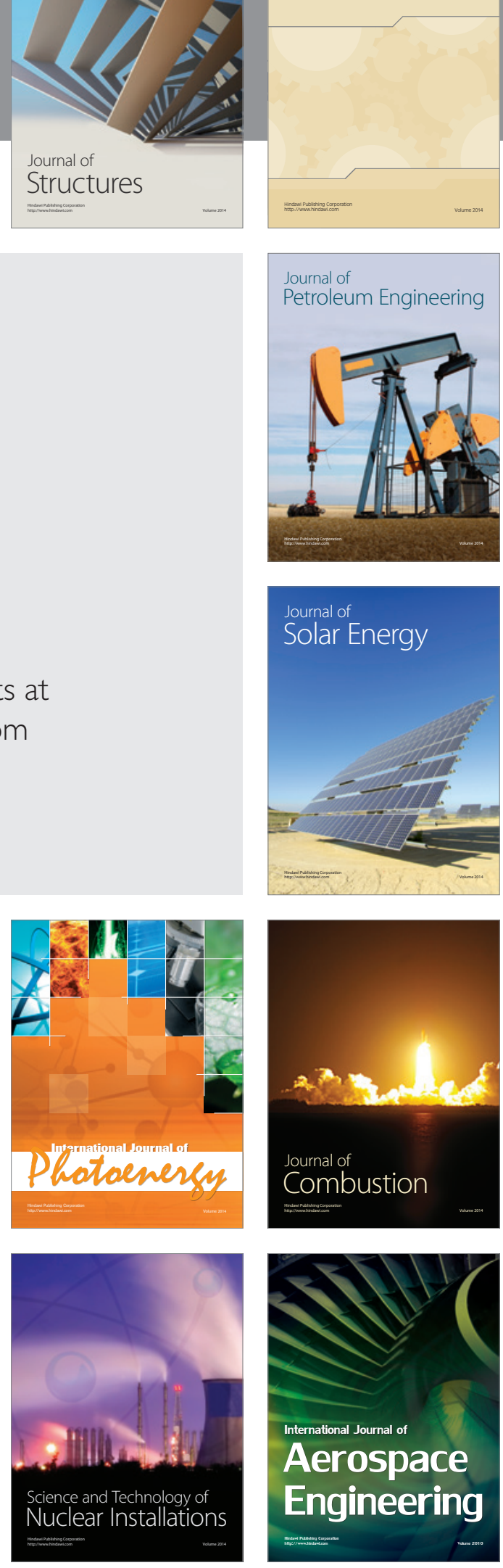